\title{
Proapoptotische Strategien gegen Melanomzellen
}

\author{
Proapoptotic Strategies against Melanoma Cells
}

Autoren

Institut
J. Eberle, B. M. Kurbanov

Charité - Universitätsmedizin Berlin

Klinik für Dermatologie, Venerologie und Allergologie

HTCC - Haut Tumor Centrum Charité

\section{Bibliografie}

DOI $10.1055 / \mathrm{s}-2006-944873$

Akt Dermatol 2006; 32;

474-480 @ Georg Thieme

Verlag KG Stuttgart · New York ISSN 0340-2541

Korrespondenzadresse PD Dr. rer. nat. Jürgen Eberle Charité - Universitätsmedizin Berlin

Klinik für Dermatologie,

Venerologie und Allergologie

HTCC - Haut Tumor Centrum

Charité

Campus Benjamin Franklin

Hindenburgdamm 30

12203 Berlin

juergen.eberle@charite.de

\section{Zusammenfassung \\ $\nabla$}

Die Apoptose stellt einen grundlegenden Mechanismus zur Aufrechterhaltung der zellulären Homöostase dar. Intrinsische proapoptotische Signalwege werden durch p53 induziert und greifen insbesondere auf der Ebene der Mitochondrien an. Demgegenüber basiert die extrinsische Induktion der Apoptose maßgeblich auf der Bindung von Todesliganden (TNF-alpha, CD95L/FasL, TRAIL) an ihre Rezeptoren sowie auf der Induktion der Caspasen-Kaskade. Für die Tumorprogression sowie für die Therapieresistenz maligner Tumoren ist die Inaktivierung proapoptotischer Signalwege eine notwendige Voraussetzung. So wurden auch verschiedene Mechanismen der Apoptoseresistenz für das im hohen Maße therapieresistente maligne Melanom identifiziert. Ziel verschiedener neuer Therapiekonzepte gegen Krebs ist daher insbesondere die

\section{Apoptose als ein Kontrollmechanismus der zellulären Homöostase $\nabla$}

Für die Funktion des menschlichen Organismus ist die umfassende Regulation der Aktivitäten seiner Billionen von Einzelzellen eine unabdingbare Voraussetzung. Dabei wirkt eine noch unüberschaubare Zahl an molekularen Regulatoren mit, wie Zytokine, Rezeptoren, Kinasen, Signalproteine und Transkriptionsfaktoren. Insbesondere geht es um die Regulation von Zellteilung, Differenzierung und der Apoptose, dem programmierten Zelltod. Die Apoptose ist somit ein elementarer Prozess in der Aufrechterhaltung der zellulären Homöostase [46].

Die besondere Morphologie der Apoptose und ihre prinzipielle Unterscheidung von der Nekrose, dem „Unfalltod der Zelle“, wurden erstmals 1972 beschrieben [32]. Die morphologischen Veränderungen betreffen die Chromatinkonden-
Überwindung dieser Apoptoseresistenz, wobei Todesliganden wichtige Hoffnungsträger darstellen. Insbesondere TRAIL (TNF-related apoptosisinducing ligand) hat aufgrund seiner hohen selektiven Effektivität gegen Tumorzellen bereits Eingang in klinische Studien gefunden. Das maligne Melanom war hierbei allerdings bisher weitgehend ausgeschlossen. TRAIL bindet an die zwei agonistischen Todesrezeptoren TRAILR1/DR4 und TRAIL-R2/DR5. Neue Befunde an Melanomzelllinien und Biopsien von Melanom-Primärtumoren zeigten, dass TRAIL in Melanomzellen über DR4 sehr effizient Apoptose induzieren kann und dass die Mehrzahl der Primärmelanome den DR4 stark exprimieren. Apoptose-gestützte Strategien stellen einen wichtigen Schritt zur Entwicklung effektiver Tumortherapien dar, und TRAIL könnte sich zu einer viel versprechenden Therapieoption auch beim malignen Melanom entwickeln.

sation, Zellschrumpfung und die Bildung apoptotischer, von Membranen umschlossener Zellfragmente (apoptotic bodies). Darüber hinaus sind eine Reihe von charakteristischen biochemischen Veränderungen zu verzeichnen, darunter die DNA-Fragmentierung. Demgegenüber bleibt die Membran-Integrität erhalten.

Auf verschiedene exogene und endogene Signale stellt die Apoptose die adäquate physiologische Antwort dar. Insbesondere auch Krebszellen sind diversen proapoptotischen Signalen ausgesetzt. Diese entstehen einerseits aufgrund von vielfältigen zellulären Fehlregulationen und chromosomalen Aberrationen in den Krebszellen sowie andererseits aufgrund des Angriffs zytotoxischer T-Lymphozyten im Rahmen der Immunantwort gegen den Tumor [24]. Daher ist die Inaktivierung apoptotischer Signalwege als eine notwendige Voraussetzung für die Tumorprogression anzusehen. Während zelluläre Schäden, auch 


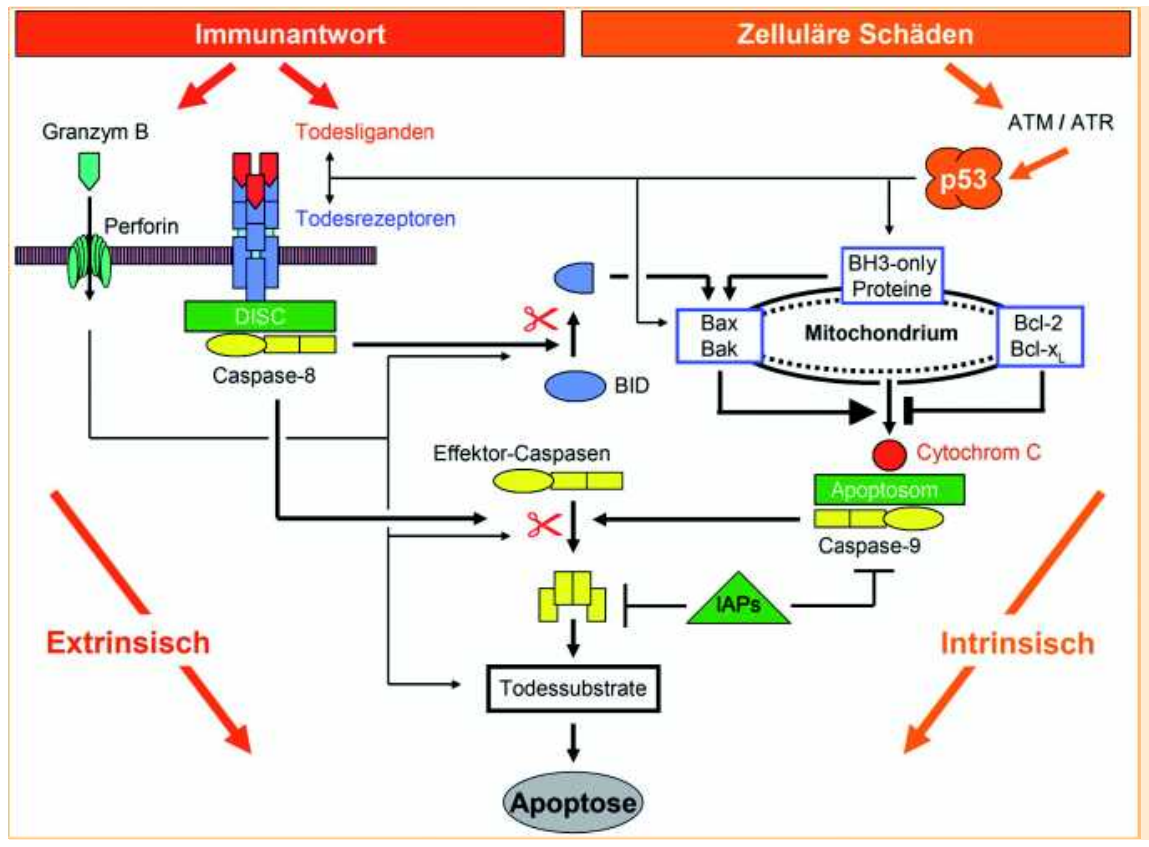

Abb. 1 Proapoptotische Signalwege. DISC: death-inducing signaling complex; IAPs: Inhibitoren der Apoptose. Weitere Erläuterungen im Text.

solche nach Chemotherapie, vornehmlich intrinsische Apoptosewege über p53 aktivieren [20], werden durch Immunzellen vornehmlich extrinsische Apoptosewege über die Todesliganden CD95L, TRAIL und TNF- $\alpha$ sowie über die Protease Granzyme B aktiviert [49].

\section{Signalwege der Apoptose}

Proapoptotische Signalwege besitzen weit reichende Konsequenzen für das Schicksal der Einzelzellen und das betroffene Gewebe und sind daher mehrfach abgesichert und gegenreguliert ( $\bullet$ Abb. 1). Gerade diese für das Überleben der normalen Zellen notwendige Option zur Gegenregulation beinhaltet vielfältige Möglichkeiten für Tumorzellen, der Apoptose zu entkommen.

Intrinsische proapoptotische Signalwege greifen auf der Ebene der Mitochondrien an. Nach Auftreten von zellulären Schäden werden verschiedene Kinasen wie ATM (ataxia telangiectasiamutated) und ATR (ATM- and rad3-related) aktiviert. Die Phosphorylierung des Transkriptionsfaktors p53 durch diese Kinasen führt zu seiner Stabilisierung und zur Transkriptionsaktivierung verschiedener Gene, darunter proapoptotische Bcl-2-Proteine (B-cell lymphoma 2) wie Bax, Bik/Nbk, Noxa und PUMA [20]. Diese sind als Gegenspieler zu den antiapoptotischen Bcl-2-Proteinen wie $\mathrm{Bcl}-2$ und $\mathrm{Bcl}-\mathrm{x}_{\mathrm{L}} \mathrm{zu}$ sehen und induzieren die Freisetzung von mitochondrialen Proteinen ins Zytosol [5]. Das dabei freigesetzte Cytochrom $C$ initiiert im Zytosol die Bildung eines Multiproteinkomplexes (das Apoptosom), in dem die Initiatorcaspase-9 aktiviert wird. Caspasen (Aspartat-spezifische CysteinProteasen) spalten ihre Zielproteine jeweils nach der Aminosäure Aspartat und führen grundlegende Funktionen in der Regulation der Apoptose aus [39].

Charakteristisch für die extrinsische Induktion der Apoptose durch zytotoxische T-Lymphozyten und NK-Zellen ist die Sezernierung der Protease Granzyme B sowie der „Todesliganden“ TNF- $\alpha$ (Tumornekrosefaktor), CD95L (CD95/Fas-Ligand) und TRAIL (TNF-related apoptosis-inducing ligand). Ihre Bindung an „Todesrezeptoren“ führt zur Rezeptor-Oligomerisierung und
Ausbildung eines membrangebundenen Proteinkomplexes (DISC: death-inducing signaling complex) unter Einbeziehung von FADD (Fas-associated death domain) und der Initiationscaspase-8. Durch das Zusammenbringen der Caspase-Moleküle in räumlicher Nähe (induced proximity) oder durch autokatalytische Proteolyse wird hier die Caspase-8 aktiviert [43].

Zur Familie der Todesrezeptoren, einer Unterfamilie der TNF-Rezeptoren, gehören TNF-Rezeptor I, CD95/Fas, die TRAIL-Rezeptoren I und II (DR4, DR5) sowie die Todesrezeptoren 3 und 6 (DR3/TRAMP; DR6; Abb. 2). Querverbindungen zwischen extrinsischen und intrinsischen proapoptotischen Signalwegen bestehen in der Aktivierung der Transkription von Todesliganden und ihren Rezeptoren durch p53 sowie in der durch Caspase-8 vermittelten Spaltung und Aktivierung des proapoptotischen Bcl-2-Proteins Bid. Dieses wechselt darauf in die mitochondriale Membran und aktiviert dort über Bax und Bak den mitochondrialen Signalweg.

In der Endphase konvergieren beide Signalwege, wobei die aktivierten Initiatorcaspasen -8 und -9 nun Effektorcaspasen wie die Caspase-3, -6 und -7 durch proteolytische Spaltung aktivieren können. Ziele der Effektorcaspasen sind eine große Zahl an zellulären Proteinen (Todessubstrate), darunter DNAsen, DNA-Reparaturenzyme und diverse Struktur- wie Signalmoleküle, was schließlich die Apoptose unwiderruflich ins Werk setzt [12]. Die von zytotoxischen T-Lymphozyten sezernierte Protease Granzyme B greift auf mehreren der hier dargestellten Schritte an und führt so zur Apoptoseinduktion in den Zielzellen. So wurde gezeigt, dass das durch Mithilfe von Perforin in die Zielzelle gelangende Granzyme B ähnlich den Caspasen sowohl Bid als auch Caspase-3 und ICAD spalten kann [49].

\section{Apoptoseresistenz von Melanomzellen $\nabla$}

Das maligne Melanom stellt aufgrund seiner ausgeprägten Therapieresistenz, der in den letzten Jahrzehnten stetig angestiegenen Inzidenz und seiner ungebrochen hohen Mortalität eine der größten Herausforderungen für den Dermatologen dar. Tumorzellen zeichnen sich durch vielfache genetische Veränderungen 


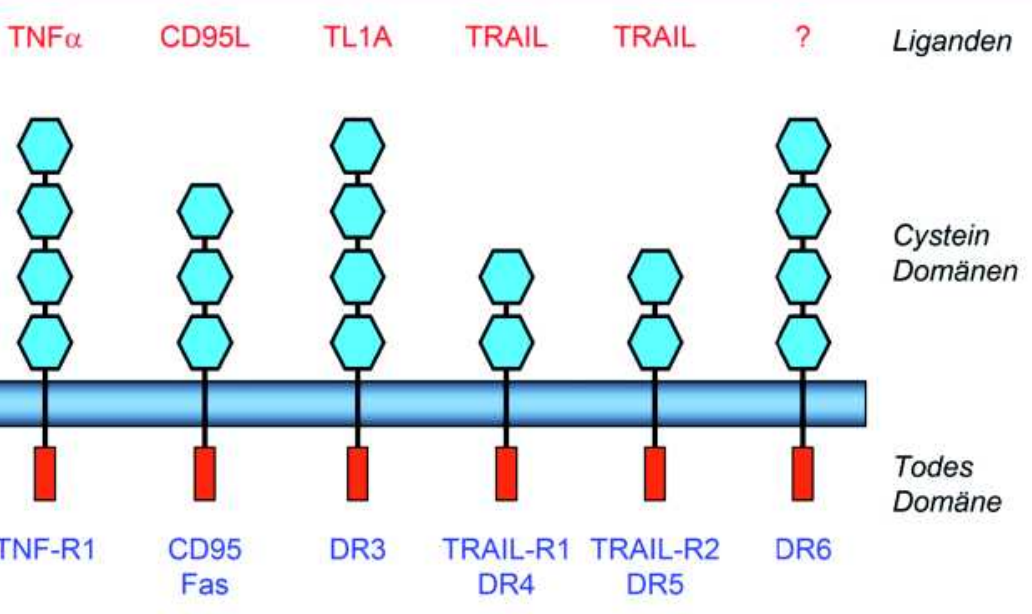

Abb. 2 Todesrezeptoren und ihre Liganden. Dargestellt sind sechs bekannte Todesrezeptoren mit ihrer Proteindomänenstruktur (extrazelluläre Cysteindomänen, Transmembrandomäne und intrazelluläre Todesdomäne) sowie darüber namentlich aufgelistet die an diese Rezeptoren bindenden Todesliganden. Der Ligand für Todesrezeptor 6 (DR6) ist noch unbekannt. An die Todesdomäne binden intrazellulär die Proteine des DISC (deathinducing signaling complex).

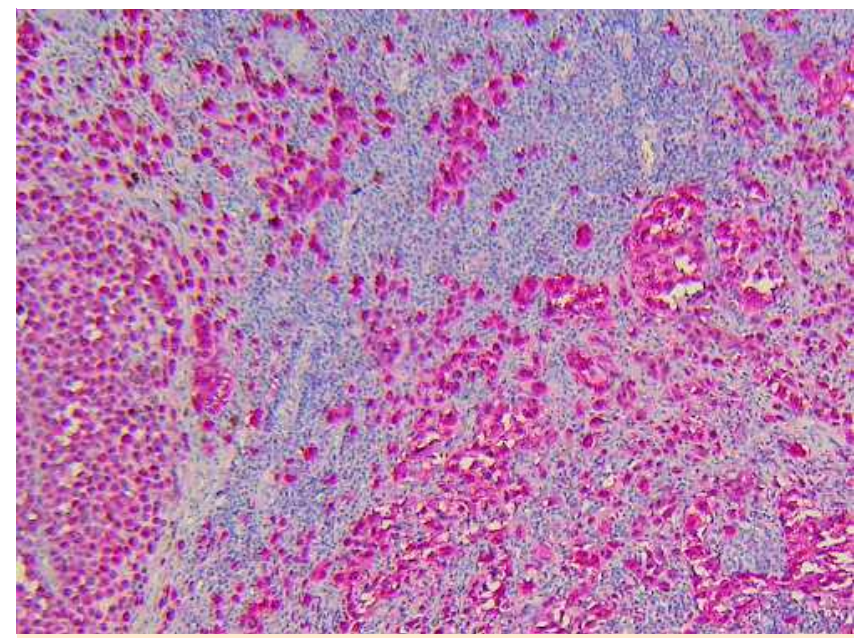

Abb. 3 Immunogenität des malignen Melanoms. Gezeigt ist ein massives lymphozytäres Tumorinfiltrat in einem malignen Melanoms nach immunhistologischer Anfärbung. Die Melanomzellen sind rot angefärbt, die kleineren Lymphozyten erscheinen grau.

und zelluläre Dysfunktionen aus, was in normalen Zellen zu einer Aktivierung der intrinsischen Apoptosewege führen sollte. Darüber hinaus besitzen die Tumorzellen veränderte Proteine, die vom Immunsystem als fremd erkannt werden können und so zur Auslösung einer Immunantwort gegen den Tumor führen können. Bezeichnenderweise ist das maligne Melanom ein immunologisch hoch reaktiver Tumor wie an den häufig zahlreichen, den Tumor infiltrierenden Lymphozyten leicht zu erkennen ist ( Abb. 3). Eine weit reichende Blockierung der Apoptose erscheint somit als notwendige Voraussetzung für das Tumorwachstum.

Grundlage der Apoptoseresistenz sind Defekte in den pro- und antiapoptotisch wirkenden Signalwegen. Bei epithelialen Tumoren stehen Mutationen des Tumorsuppressorgens p53 im Vordergrund und korrelieren mit einer ungünstigen Prognose [20]. Obwohl p53-Mutationen beim Melanom eher selten vorkommen, gibt es auch hier Indizien für eine Inaktivierung des betreffenden Signalweges $[33,50]$. Eine Blockierung des mitochondrialen Weges könnte auch auf der Ebene des Adapterproteins Apaf-1 bestehen, für das in vielen Melanom-Zelllinien ein Ver- lust der Heterozygosität (LOH) sowie DNA-Methylierung des verbliebenen Allels beschrieben wurde [55].

Die proapoptotischen Apoptosewege laufen auf der Ebene der Effektorcaspasen zusammen ( $\bullet$ Abb. 1). Hier greifen zelleigene Proteininhibitoren, IAPs (inhibitors of apoptosis proteins) an. In Melanom-Zelllinien, Melanommetastasen und auch in invasiven Primärtumoren fand sich hohe Expression von Survivin, einem Inhibitor der Effektor-Caspasen 3 und 7, [16]. Auch fand sich in einigen Melanomzellinien Expression von Livin/ML-IAP, einem Inhibitor der Caspase-9 [29,40].

Für eine Blockierung extrinsischer Signalwege spricht die in Melanomzellen und in Melanommetastasen hohe Expression von cFLIP (flice-inhibitory protein), ein kompetitiver Inhibitor der Caspase-8 [25]. Eine weitere Möglichkeit zur Blockierung der extrinsischen Signalwege liegt in der Herabregulation oder Mutation der Todesrezeptoren, was relativ häufig in hämatologischen wie in soliden Tumoren anzutreffen ist [38].

Verschiedene Arbeiten der letzten Jahre wiesen auf eine besondere Bedeutung des mitochondrialen Signalweges in Melanomzellen hin $[23,44]$. Die Bcl-2-Proteine als wichtige Regulatoren dieses Signalweges erscheinen somit von besonderer Bedeutung für die Apoptoseregulation in Melanomzellen. Typisch für Melanome ist die hohe Expression von Bcl-2 [18] sowie von $\mathrm{Bcl}-\mathrm{x}_{\mathrm{L}}$ [21]. Als kritischer Faktor für die Apoptosesensitivität von Melanomzellen erwies sich das Expressionsverhältnis von pro- zu anti-apoptotischen Bcl-2-Proteinen [45].

Schließlich gehen verschiedene antiapoptotische Programme auf die Aktivitäten der Transkriptionsfaktoren der NF-кB-Familie (nuclear factor-kappaB) zurück, die neben essentiellen proinflammatorischen Funktionen auch die Expression antiapoptotischer Faktoren aus den Familien der Bcl-2-Proteine sowie der IAPs regulieren [8].

\section{Strategien zur Überwindung der Apoptose- resistenz beim malignen Melanom $\nabla$}

Die Mehrzahl der zytotoxischen und hormonellen Therapiekonzepte sowie die Strahlentherapie zielen schließlich darauf $a b$, Krebszellen durch Induktion der Apoptose zu eliminieren. Dementsprechend kann die Inaktivierung apoptotischer Signalwege auch die Ursache für Resistenzen gegenüber Chemo- und Strahlentherapie darstellen [53]. Gerade die ausgeprägte klinische 
Chemotherapie-Resistenz des Melanoms lässt auf eine Blockierung der Apoptose-Programme in Melanomzellen schließen [56]. Ziel verschiedener neuer Therapiekonzepte gegen Krebs ist deshalb insbesondere die Überwindung der Apoptoseresistenz in den Tumorzellen $[13,46]$.

Die hohe Bcl-2-Expression in Melanomzellen war Anlass für therapeutische Strategien unter Verwendung von antisense-Molekülen gegen Bcl-2 (Oblimersen). Diese zeigten in-vitro sowie in Tierexperimenten ein hohes anti-Tumor Potential und befinden sich in der klinischen Erprobungsphase [27,34]. Erste publizierte Ergebnisse konnten allerdings bisher keine entscheidenden Verbesserungen im Krankheitsverlauf beim metastasierten Melanom belegen [13].

Komplementär zur Herabregulation von antiapoptotischen Bcl2-Proteinen ist die Überexpression von proapoptotischen Bcl2-Proteinen. Dieser Ansatz zeigte sich in vitro sowie in Tiermodellen geeignet zur effizienten Induktion von Apoptose in Melanomzellen sowie zur Sensitivierung für Chemotherapeutika $[21,22,42]$. Die wichtige Rolle des mitochondrialen Signalweges und der proapoptotischen Bcl-2-Proteine für die Apoptoseregulation in Melanomzellen wurde weiter durch immunhistologische Befunde unterstrichen, die zeigten, dass ein Verlust der proapoptotischen Bcl-2-Proteine Bax und Bak in primären Melanomen mit verschlechterten Prognose der Patienten korreliert ist [11]. Neue Strategien unter Verwendung von Peptiden sowie natürlich vorkommenden Proteinen mit Homologie zur proapoptotischen BH3-Domäne der Bcl-2-Proteine werden als mögliche Therapieoptionen diskutiert [13].

Die Bedeutung des Transkriptionsfaktors NF- $\kappa B$ für die Apoptoseresistenz von Tumorzellen ist die Basis für therapeutische Strategien unter Verwendung von Proteasom-Inhibitoren, die den Abbau des Inhibitors von NF- $\kappa B$ (I- $\kappa B$ ) verzögern [59]. Dadurch wird die durch NF- $\mathrm{kB}$ gesteuerte Expression von antiapoptotischen Faktoren unterbunden. Diese durch NF- $\mathrm{kB}$ transkriptionell aktivierten Faktoren schließen antiapoptotische Bcl-2-Proteine wie $\mathrm{Bcl}-\mathrm{x}_{\mathrm{L}}$ sowie Caspase-Inhibitoren wie cIAP-1, cIAP-2, XIAP als auch cFLIP ein [28]. Proteasom-Inhibitoren befinden sich bereits für einige Tumoren in der klinischen Erprobungsphase [47]. In vitro sowie im Mausmodell konnte der Antitumor-Effekt solcher Inhibitoren auch beim Melanom bereits demonstriert werden [1].

Schließlich gelten Todesliganden als wichtige Hoffnungsträger für künftige therapeutische Konzepte gegen Krebs. In seiner Bedeutung für die Apoptoseregulation in Melanomzellen wurde das CD95-Signalsystem bisher kontrovers diskutiert $[3,17]$. Die Überexpression von CD95L erwies sich aber als eine effiziente Strategie zur Induktion von Apoptose in Melanomzellen sowohl in vitro als auch in Melanom-Mausmodellen [9]. Insbesondere auch der Vergleich mit anderen Tumorzelllinien belegte eine besondere Sensitivität von Melanomzellen gegenüber diesem Todesliganden. Eine selektive Apoptoseinduktion in Melanomzellen konnte durch eine über den Tyrosinasepromotor gesteuerte Expression des CD95L erreicht werden, wichtige Voraussetzung für mögliche gentherapeutische Weiterentwicklungen dieses Systems [10].

\section{Therapeutische Konzepte unter Verwendung von TRAIL}

$\nabla$

Die pharmakologische, nicht selektive Applikation von CD95L erscheint allerdings aufgrund von in Tiermodellen zu beobachtenden massiven Nebenwirkungen, wie insbesondere Leber-Toxizität, derzeit nicht möglich [41]. Ähnlich ungünstig erscheint eine generalisierte, pharmakologische Verwendung des Todesliganden TNF- $\alpha$ aufgrund von massiven, durch TNF- $\alpha$ induzierten Entzündungsreaktionen [2]. Allerdings wurde TNF- $\alpha$ bei der isolierten Extremitätenperfusion für multible regionale Melanommetastasen teilweise erfolgreich eingesetzt [48]. Die Probleme der systemischen Toxizität scheinen demgegenüber für den dritten Todesliganden TRAIL nur von untergeordneter Bedeutung zu sein.

Fünf TRAIL-Rezeptoren wurden identifiziert, wobei TRAILR1/DR4 und TRAIL-R2/DR5 als agonistische Todesrezeptoren parallel zu CD95 die Caspasen-Kaskade sowie, abhängig vom Zelltyp, den mitochondrialen Signalweg, MAP-Kinasen und NF- $\mathrm{kB}$ aktivieren können [6,7]. Demgegenüber sind die TRAIL-Rezeptoren DcR1, DcR2 und OPG antagonistisch. Sie führen nicht zu einer Weiterleitung der Signalkaskakade und unterdrücken so das Todessignal. Die proapoptotische Signalkaskade schließt Rezeptor-Oligomerisierung, Bildung des DISC (death-inducing signalling complex), Aktivierung von Initiatorcaspasen, Spaltung von Effektorcaspasen und schließlich DNA-Fragmentierung ein [61].

Die besondere Signifikanz von TRAIL für therapeutische Erwägungen beruht auf seiner Eigenschaft, Apoptose bevorzugt in Tumorzellen zu induzieren, während viele nicht-maligne Zellen nicht oder weniger sensitiv auf TRAIL reagierten [37,63]. Bezeichnenderweise wird TRAIL auch von verschiedenen normalen Gewebszellen exprimiert, was nur mit einer relativ guten Verträglichkeit normaler Zellen vereinbar ist [62].

Auch in Tiermodellen resultierte die Behandlung mit TRAIL als Einzeltherapie oder in Kombination mit Chemotherapeutika in einer Suppression des Tumorwachstums ohne ersichtliche Zeichen von systemischer Toxizität. So zeigte sich das hohe Potential von TRAIL in Mäusen und in Primaten, wenn es einzeln oder in Kombination mit Chemotherapeutika verabreicht wurde. Unterdrückung des Wachstums von Tumoren in diesen Modellen ohne Hinweise auf systemische Toxizität qualifizierten TRAIL als einen vielversprechenden Kandidaten für Tumortherapien $[37,51,60]$. Darüber hinaus belegten Experimente mit TRAIL-defizienten Mäusen (knockout) seine tragende Rolle auch in der körpereigenen Tumorabwehr durch Immunzellen [4,54].

Zur selektiven Aktivierung jeweils eines einzelnen der zwei agonistischen TRAIL-Rezeptoren wurden rekombinante TRAIL-Derivate sowie agonistische monoklonale DR4- und DR5-Antikörper entwickelt und als vergleichbar effizient in Mäusen getestet [31,58]. Seine Selektivität gegenüber Tumorzellen ermutigten zu verschiedenen klinischen Studien unter Verwendung von TRAIL oder von agonistischen Antikörpern gegen TRAIL-Rezeptoren bei Patienten mit Lungenkrebs, Non-Hodgkin-Lymphom und kolorektalem Karzinom, deren Ergebnis mit großer Hoffnung erwartet wird [13]. 


\section{TRAIL-Sensitivität und Expression der TRAIL- Rezeptoren in Melanomzellen \\ $\nabla$}

Die Verwendung von TRAIL als Anti-Tumor-Therapie beruht auf der Funktionalität der entsprechenden agonistischen Rezeptoren. Entgegen den Befunden bei epithelialen Tumoren mit vorherrschender Rolle des DR4 $[30,57]$ wurde bisher für Melanomzellen angenommen, dass ihre Sensitivität für TRAIL insbesondere von der Aktivität des DR5 abhängt. Die biologische Signifikanz des DR4 wurde dagegen vernachlässigt $[19,65]$.

Unsere Untersuchungen an Melanomzellinien zeigten dem gegenüber, dass DR4 ein entscheidender Regulator für die TRAILSensitivität in Melanomzellen ist. Während DR5 konsistent in Melanom-Zellinien exprimiert war, fand sich signifikante Expression von DR4 zwar nur in nur 2/7 Zelllinien, doch zeichneten sich die DR4-positiven Melanomzellen durch hohe Sensitivität gegenüber TRAIL-induzierter Apoptose aus. Demgegenüber zeigten DR5-Zellen (DR4-negativ) eine verminderte und zeitlich verzögerte Antwort (2/7 Zelllinien) oder waren komplett resistent (3/7 Zelllinien). Die Verwendung von selektiven DR4/DR5 blockierenden Antikörpern belegte zweifelsfrei die vorherrschende Rolle des DR4 auch in den Melanomzellen, die beide Rezeptoren exprimierten [36].

Die Bedeutung dieser Befunde für die In-vivo-Situation zeigte sich aufgrund von immunhistochemischen Untersuchungen. Diese belegten erstmals eine signifikante Expression beider Rezeptoren in der überwiegenden Mehrzahl von Primärmelanomen. Diese Befunde lassen auf eine hohe Eignung von TRAIL auch für therapeutische Strategien beim Melanom hoffen [36].

\section{Ursachen für Resistenzen gegen TRAIL}

$\nabla$

Allerdings erscheint das relativ häufige Auftreten von Resistenzen gegen TRAIL problematisch für die Entwicklung effizienter Therapien [61]. Als Ursachen für die Resistenz gegen TRAIL wurden verstärkte Expression von antiapoptotischen Faktoren wie c-FLIP, Bcl-2, Bcl- $\mathrm{x}_{\mathrm{L}}$ und IAPs (inhibitors of apoptosis proteins) vorgeschlagen [64]. Da der Transkriptionsfaktor NF- $\kappa B$ von TRAIL aktiviert wird und seinerseits die Transkription verschiedener dieser antiapoptotischen Proteine steuert, wurde eine entscheidende Rolle von NF-kB für die Resistenz gegen TRAIL angenommen $[14,26]$.

Unsere Untersuchungen zeigten jedoch in Melanomzellen keinen direkten Zusammenhang zwischen TRAIL-Resistenz und der durch TRAIL induzierten Aktivierung von NF- $\kappa B$ sowie der Expression antiapoptotischer Faktoren. Vielmehr ergaben sich in einem Melanom-Zellkulturmodell für TRAIL-Resistenz deutliche Hinweise auf eine Bedeutung der Herabregulation von proapoptotischen Faktoren wie den Initiatorcaspasen und des DR4 für die TRAIL-Resistenz.

\section{Apoptose-gestiutze Therapien \\ $\nabla$}

Derzeit wird eine große Zahl von neuen therapeutischen Konzepten entwickelt und befindet sich auf der Stufe der klinischen Anwendung. Diese neuen Konzepte schließen verschiedene proapoptotische Strategien ein $[13,46]$. Neben den vorgenannten Strategien der Blockierung der Bcl-2-Expression und der Blockierung der NF-кB-Aktivierung sind hier insbesondere die Untersuchungen mit TRAIL relativ weit gediehen.
Aufgrund früherer Einschätzungen erschien allerdings die Verwendung von TRAIL beim Melanom eher weniger viel versprechend. Dies beruhte insbesondere auf einer Unterschätzung der Bedeutung des DR4 für die TRAIL-induzierte Apoptose in Melanomzellen. Die kürzlich erhobenen Befunde der Expression von DR4 in Primärmelanomen und der hohen Effizienz der über DR4 vermittelten Apoptose in Melanomzellen lassen TRAIL und insbesondere auf DR4 basierende Strategien als besonders aussichtsreich mit Hinblick auf neue Therapieansätze beim Melanom erscheinen.

Einer TRAIL-Resistenz auf der Basis einer Herabregulation des DR4 oder der Initiatorcaspasen könnte mit kombinierten Behandlungsstrategien begegnet werden. So wurde gezeigt, dass die TRAIL-Todesrezeptoren nach Chemotherapie hoch reguliert werden [52], während die Expression von Caspasen durch Interferon- $\gamma$ gesteigert werden konnte [15]. Weitere, intensive Forschung wird nötig sein, um geeignete Bedingungen zu entwickeln, die schließlich erfolgreiche therapeutische Ansätze für das maligne Melanom auf der Basis von TRAIL oder von anderen proapoptotischen Faktoren ermöglichen. Erste Erfolge sprechen aber dafür, dass Apoptose-gestützte Strategien einen wichtigen Schritt zur Entwicklung effektiver Tumortherapien darstellen können.

\section{Danksagung \\ $\nabla$}

Die Arbeit wurde gefördert durch die Berliner Stiftung für Dermatologie, die Deutsche Krebshilfe und die Islamische Bank für Entwicklung.

\section{Abstract}

\section{Proapoptotic Strategies against Melanoma Cells $\nabla$}

Apoptosis represents a basic mechanism for maintenance of cellular homeostasis. Intrinsic proapoptotic pathways are induced by p53 and employ especially the mitochondrial level. In contrast, the extrinsic induction of apoptosis is based on the binding of death ligands (TNF-alpha, CD95L/FasL, TRAIL) to their respective receptors and on induction of the caspase cascade. For tumor progression and for therapy resistance of malignant tumors, the inactivation of proapoptotic pathways is a prerequisite. Thus, several mechanisms of apoptosis resistance have been identified for the highly therapy resistant malignant melanoma. Therefore many new anti-cancer concepts aim especially to overcome this apoptosis resistance, and death ligands may supply promising strategies. Especially with TRAIL (TNF-related apoptosis-inducing ligand) several clinical studies have been initiated because of its high and selective efficiency against tumor cells. However, these studies so far largely excluded melanoma. TRAIL binds the two agonistic death receptors TRAIL-R1/DR4 and TRAIL-R2/DR5. New data on melanoma cell lines and biopsies revealed that TRAIL can efficiently induce apoptosis in melanoma cells via DR4, and the majority of primary melanomas do express DR4. Apoptosis-based strategies represent an important step in development of tumor therapies, and TRAIL may evolve into a promising therapeutic option also for melanoma. 


\section{Literatur}

1 Amiri KI, Horton LW, LaFleur BJ, Sosman JA, Richmond A. Augmenting chemosensitivity of malignant melanoma tumors via proteasome inhibition: implication for bortezomib (VELCADE, PS-341) as a therapeutic agent for malignant melanoma. Cancer Res 2004; 64: 49124918

2 Ashkenazi A. Targeting death and decoy receptors of the tumour-necrosis factor superfamily. Nat Rev Cancer 2002; 2: 420-430

3 Chappell DB, Zaks TZ, Rosenberg SA, Restifo NP. Human melanoma cells do not express Fas (Apo-1/CD95) ligand. Cancer Res 1999; 59: 59-62

4 Cretney E, Takeda K, Yagita H, Glaccum M, Peschon IJ, Smyth MJ. Increased susceptibility to tumor initiation and metastasis in TNF-related apoptosis-inducing ligand-deficient mice. J Immunol 2002; 168: $1356-1361$

5 Daniel PT, Schulze-Osthoff K, Belka C, Guner D. Guardians of cell death: the Bcl-2 family proteins. Essays Biochem 2003; 39: $73-88$

6 Daniel PT, Wieder T, Sturm I, Schulze-Osthoff K. The kiss of death: promises and failures of death receptors and ligands in cancer therapy. Leukemia 2001; 15: 1022 - 1032

7 Di Pietro R, Zauli G. Emerging non-apoptotic functions of tumor necrosis factor-related apoptosis-inducing ligand (TRAIL)/Apo2L. J Cell Physiol 2004; 201: 331 - 340

8 Dolcet X, Llobet D, Pallares J, Matias-Guiu X. NF-kB in development and progression of human cancer. Virchows Arch 2005; 446: 475- 482

9 Eberle J, Fecker LF, Hossini AM, Wieder T, Daniel PT, Orfanos CE, Geilen CC. CD95/Fas signaling in human melanoma cells: conditional expression of CD95L/FasL overcomes the intrinsic apoptosis resistance of malignant melanoma and inhibits growth and progression of human melanoma xenotransplants. Oncogene 2003; 22: 9131 - 9141

10 Fecker LF, Geilen CC, Hossini AM, Schwarz C, Fechner H, Bartlett DL, Orfanos $C E$, Eberle J. Selective induction of apoptosis in melanoma cells by tyrosinase promoter-controlled CD95 ligand overexpression. J Invest Dermatol 2005; 124: 221 - 228

11 Fecker LF, Geilen CC, Tchernev G, Trefzer U, Assaf C, Kurbanov BM, Schwarz C, Daniel PT, Eberle J. Loss of proapoptotic Bcl-2-related multidomain proteins in primary melanomas is associated with poor prognosis. J Invest Dermatol 2006; 126: 1366 - 1371

12 Fischer U, Janicke RU, Schulze-Osthoff K. Many cuts to ruin: a comprehensive update of caspase substrates. Cell Death Differ 2003; 10: 76 100

13 Fischer U, Schulze-Osthoff K. Apoptosis-based therapies and drug targets. Cell Death Differ 2005; 12: 942 - 961

14 Franco AV, Zhang XD, Van Berkel E, Sanders JE, Zhang XY, Thomas WD, Nguyen T, Hersey P. The role of NF-kappa B in TNF-related apoptosisinducing ligand (TRAIL)-induced apoptosis of melanoma cells. J Immunol 2001; 166: 5337-5345

15 Fulda S, Debatin KM. 5-Aza-2"-deoxycytidine and IFN-gamma cooperate to sensitize for TRAIL-induced apoptosis by upregulating caspase-8. Oncogene 2006; Epub ahead of print

16 Grossman D, McNiff JM, Li F, Altieri DC. Expression and targeting of the apoptosis inhibitor, survivin, in human melanoma. J Invest Dermatol 1999; 113: 1076- 1081

17 Hahne $M$, Rimoldi $D$, Schroter $M$, Romero $P$, Schreier $M$, French $L E$, Schneider P, Bornand T, Fontana A, Lienard D, Cerottini J, Tschopp J. Melanoma cell expression of Fas(Apo-1/CD95) ligand: implications for tumor immune escape. Science 1996; 274: 1363-1366

18 Hakansson A, Gustafsson B, Abdiu A, Krysander L, Hakansson L. Bcl-2 expression in metastatic malignant melanoma. Importance for the therapeutic efficacy of biochemotherapy. Cancer Immunol Immunother 2003; 52: $249-254$

19 Hersey P, Zhang XD. How melanoma cells evade trail-induced apoptosis. Nat Rev Cancer 2001; 1: $142-150$

20 Hofseth LJ, Hussain SP, Harris CC. p53:25 years after its discovery. Trends Pharmacol Sci 2004; 25: 177-181

21 Hossini AM, Eberle J, Fecker LF, Orfanos CE, Geilen CC. Conditional expression of exogenous Bcl-X(S) triggers apoptosis in human melanoma cells in vitro and delays growth of melanoma xenografts. FEBS Lett 2003; 553: $250-256$

22 Hossini AM, Geilen CC, Fecker LF, Daniel PT, Eberle J. A novel Bcl-x splice product, Bcl-xAK, triggers apoptosis in human melanoma cells without BH3 domain. Oncogene 2006; 25: 2160 - 2169

23 Hussein MR, Haemel AK, Wood GS. Apoptosis and melanoma: molecular mechanisms. J Pathol 2003; 199: 275-288

24 Igney FH, Krammer PH. Death and anti-death: tumour resistance to apoptosis. Nat Rev Cancer 2002; 2: 277-288
25 Irmler M, Thome M, Hahne M, Schneider P, Hofmann K, Steiner V, Bodmer JL, Schroter M, Burns K, Mattmann C, Rimoldi D, French LE, Tschopp J. Inhibition of death receptor signals by cellular FLIP. Nature 1997; 388: $190-195$

26 Ivanov VN, Bhoumik A, Ronai Z. Death receptors and melanoma resistance to apoptosis. Oncogene 2003; 22: $3152-3161$

27 Jansen B, Schlagbauer-Wadl H, Brown BD, Bryan RN, van Elsas A, Muller $M$, Wolff K, Eichler HG, Pehamberger $\mathrm{H}$. bcl-2 antisense therapy chemosensitizes human melanoma in SCID mice. Nat Med 1998; 4: $232-$ 234

28 Karin M, Greten FR. NF-kappaB: linking inflammation and immunity to cancer development and progression. Nat Rev Immunol 2005; 5: $749-759$

29 Kasof GM, Gomes BC. Livin, a novel inhibitor of apoptosis protein family member. J Biol Chem 2001; 276: 3238 - 3246

30 Kazhdan I, Marciniak RA. Death receptor 4(DR4) efficiently kills breast cancer cells irrespective of their sensitivity to tumor necrosis factorrelated apoptosis-inducing ligand (TRAIL). Cancer Gene Ther 2004; 11: $691-698$

31 Kelley RF, Totpal K, Lindstrom SH, Mathieu M, Billeci K, Deforge L, Pai R, Hymowitz SG, Ashkenazi A. Receptor-selective mutants of apoptosisinducing ligand 2 /tumor necrosis factor-related apoptosis-inducing ligand reveal a greater contribution of death receptor (DR) 5 than DR4 to apoptosis signaling. J Biol Chem 2005; 280: 2205 - 2212

32 Kerr JF, Wyllie AH, Currie AR. Apoptosis: a basic biological phenomenon with wide-ranging implications in tissue kinetics. Br J Cancer 1972; 26: 239-257

33 Kichina JV, Rauth S, Das Gupta TK, Gudkov AV. Melanoma cells can tolerate high levels of transcriptionally active endogenous p53 but are sensitive to retrovirus-transduced p53. Oncogene 2003; 22: 4911 4917

34 Klasa RJ, Gillum AM, Klem RE, Frankel SR. Oblimersen Bcl-2 antisense: facilitating apoptosis in anticancer treatment. Antisense Nucleic Acid Drug Dev 2002; 12: 193 - 213

35 Kurbanov BM, Fecker LF, Geilen CC, Sterry W, Eberle J. Resistance of melanoma cells to TRAIL does not result from upregulation of antiapoptotic proteins by NF-kB but is related to downregulation of initiator caspases and DR4. Submitted 2006

36 Kurbanov BM, Geilen CC, Fecker LF, Orfanos CE, Eberle J. Efficient TRAILR1/DR4-mediated apoptosis in melanoma cells by tumor necrosis factor-related apoptosis-inducing ligand (TRAIL). J Invest Dermatol 2005; 125: $1010-1019$

37 LeBlanc HN, Ashkenazi A. Apo2L/TRAIL and its death and decoy receptors. Cell Death Differ 2003; 10: 66 - 75

$38 \mathrm{Li}$-Weber M, Krammer PH. Function and regulation of the CD95 (APO1/Fas) ligand in the immune system. Semin Immunol 2003; 15: 145 157

39 Los M, Stroh C, Janicke RU, Engels IH, Schulze-Osthoff K. Caspases: more than just killers? Trends Immunol 2001; 22: 31 -34

40 Nachmias B, Ashhab Y, Bucholtz V, Drize O, Kadouri L, Lotem M, Peretz T, Mandelboim O, Ben Yehuda D. Caspase-mediated cleavage converts Livin from an antiapoptotic to a proapoptotic factor: implications for drug-resistant melanoma. Cancer Res 2003; 63: 6340-6349

41 Ogasawara J, Watanabe-Fukunaga R, Adachi M, Matsuzawa A, Kasugai T, Kitamura Y, Itoh N, Suda T, Nagata S. Lethal effect of the anti-Fas antibody in mice. Nature 1993; 364: 806-809

42 Oppermann M, Geilen CC, Fecker LF, Gillissen B, Daniel PT, Eberle J. Caspase-independent induction of apoptosis in human melanoma cells by the proapoptotic Bcl-2-related protein Nbk/Bik. Oncogene 2005; 24: $7369-7380$

43 Peter ME, Krammer PH. The CD95(APO-1/Fas) DISC and beyond. Cell Death Differ 2003; 10: 26-35

44 Raisova M, Bektas M, Wieder T, Daniel P, Eberle J, Orfanos CE, Geilen CC. Resistance to CD95/Fas-induced and ceramide-mediated apoptosis of human melanoma cells is caused by a defective mitochondrial cytochrome c release. FEBS Lett 2000; 473: 27-32

45 Raisova M, Hossini AM, Eberle J, Riebeling C, Wieder T, Sturm I, Daniel PT, Orfanos CE, Geilen CC. The Bax/Bcl-2 ratio determines the susceptibility of human melanoma cells to CD95/Fas-mediated apoptosis. J Invest Dermatol 2001; 117: 333 - 340

46 Reed JC, Pellecchia M. Apoptosis-based therapies for hematologic malignancies. Blood 2005; 106: 408 - 418

47 Richardson PG, Briemberg H, Jagannath S, Wen PY, Barlogie B, Berenson J, Singhal S, Siegel DS, Irwin D, Schuster M, Srkalovic G, Alexanian R, Rajkumar SV, Limentani S, Alsina M, Orlowski RZ, Najarian K, Esseltine 
$D$, Anderson KC, Amato AA. Frequency, characteristics, and reversibility of peripheral neuropathy during treatment of advanced multiple myeloma with bortezomib. J Clin Oncol 2006; 24: 3113-3120

48 Rossi CR, Foletto M, Mocellin S, Pilati PL, Campana L, Ribello D, Lise M. TNF-based limb perfusion for cutaneous melanoma in transit metastases: suggestions for modification of the perfusional schedule. J Exp Clin Cancer Res 2003; 22: $103-107$

49 Russell JH, Ley TJ. Lymphocyte-mediated cytotoxicity. Annu Rev Immunol 2002; 20: 323-370

50 Satyamoorthy K, Chehab NH, Waterman MJ, Lien MC, El Deiry WS, Herlyn $M$, Halazonetis TD. Aberrant regulation and function of wild-type p53 in radioresistant melanoma cells. Cell Growth Differ 2000; 11: $467-474$

51 Shankar S, Chen X, Srivastava RK. Effects of sequential treatments with chemotherapeutic drugs followed by TRAIL on prostate cancer in vitro and in vivo. Prostate 2005; 62: 165 - 186

52 Singh TR, Shankar S, Chen X, Asim M, Srivastava RK. Synergistic interactions of chemotherapeutic drugs and tumor necrosis factor-related apoptosis-inducing ligand/Apo-2 ligand on apoptosis and on regression of breast carcinoma in vivo. Cancer Res 2003; 63: 5390 - 5400

53 Sjostrom J, Bergh J. How apoptosis is regulated, and what goes wrong in cancer. BMJ 2001; 322: 1538 - 1539

54 Smyth MJ, Takeda K, Hayakawa Y, Peschon JJ, van den Brink MR, Yagita $H$. Nature's TRAIL - on a path to cancer immunotherapy. Immunity 2003; 18: 1-6

55 Soengas MS, Capodieci P, Polsky D, Mora J, Esteller M, Opitz-Araya X, McCombie R, Herman JG, Gerald WL, Lazebnik YA, Cordon-Cardo C, Lowe SW. Inactivation of the apoptosis effector Apaf-1 in malignant melanoma. Nature 2001; 409: 207-211

56 Soengas MS, Lowe SW. Apoptosis and melanoma chemoresistance. Oncogene 2003; 22: $3138-3151$

57 Strater J, Hinz U, Walczak H, Mechtersheimer G, Koretz K, Herfarth C, Moller P, Lehnert T. Expression of TRAIL and TRAIL receptors in colon carcinoma: TRAIL-R1 is an independent prognostic parameter. Clin Cancer Res 2002; 8: $3734-3740$

58 Takeda K, Yamaguchi N, Akiba H, Kojima Y, Hayakawa Y, Tanner JE, Sayers TJ, Seki N, Okumura K, Yagita H, Smyth MJ. Induction of tumor-specific T cell immunity by anti-DR5 antibody therapy. J Exp Med 2004; 199: $437-448$

59 Voorhees PM, Dees EC, O'Neil B, Orlowski RZ. The proteasome as a target for cancer therapy. Clin Cancer Res 2003; 9: 6316-6325

60 Walczak H, Miller RE, Ariail K, Gliniak B, Griffith TS, Kubin M, Chin W, Jones J, Woodward A, Le T, Smith C, Smolak P, Goodwin RG, Rauch CT, Schuh JC, Lynch DH. Tumoricidal activity of tumor necrosis factor-related apoptosis-inducing ligand in vivo. Nat Med 1999; 5: 157-163

61 Wang S, El Deiry WS. TRAIL and apoptosis induction by TNF-family death receptors. Oncogene 2003; 22: 8628-8633

62 Wiley SR, Schooley K, Smolak PJ, Din WS, Huang CP, Nicholl JK, Sutherland GR, Smith TD, Rauch C, Smith CA. Identification and characterization of a new member of the TNF family that induces apoptosis. Immunity $1995 ; 3: 673-682$

63 Yagita H, Takeda K, Hayakawa Y, Smyth MJ, Okumura K. TRAIL and its receptors as targets for cancer therapy. Cancer Sci 2004; 95: 777 - 783

64 Zhang L, Fang B. Mechanisms of resistance to TRAIL-induced apoptosis in cancer. Cancer Gene Ther 2005; 12: 228 - 237

65 Zhang XY, Zhang XD, Borrow JM, Nguyen T, Hersey P. Translational control of tumor necrosis factor-related apoptosis-inducing ligand death receptor expression in melanoma cells. J Biol Chem 2004; 279: $10606-10614$

\section{Buchbesprechung}

Atlas der Dermatologie (DVD-ROM)

T. L. Diepgen, M. Simon, A. Bittdorf, M. Fartasch, G. Schuler

Berlin: Springer, 2005 (in Englisch, Deutsch, Spanisch). 49,95

ISBN 3-540-00043-7

Die Autorengemeinschaft Diepgen, Simon, Bittdorf, Fartasch und Schuler legen die zweite Auflage der DVD-ROM vor, mit der wahlweise in Englisch, Deutsch oder Spanisch gearbeitet werden kann. Die Atlas CD-ROM funktioniert mit den üblichen Betriebssystemen, wenn ein WebBrowser installiert ist; falls nicht, kann er von der CD-ROM geladen werden. Nach der Sprachentscheidung gelangt man auf eine geführte Tour, auf der sich die Drei-Gliederung in alphabetisch geordnete Diagnosen, Lokalisation der Läsionen und Quiz erschließt. 600 Diagnosen stehen zur Verfügung, zu fast allen sind mehrere Bilder hinterlegt, insgesamt 4000. Beim Arbeiten im Abschnitt Lokalisation finden sich dann auch seltene Diagnosen, die im alphabetischen Diagnosenverzeichnis nicht gelistet sind. Im Quiz kann nicht nur die Diagnose aufgelöst werden, es kann auch zu einem Informationskasten mit Erläuterungen zur Diagnose gesprungen werden. Differenzialdiagnosen werden angeboten. Bilddetails können per Zoom vergrößert werden. Die Bilder haben gute Qualität, die Bibliographie ist kurz gehalten, die Handhabung gut zu verstehen und die Abfolge der Bilder oder Textkästen erfolgt schnell.

Zielgruppe ist sicherlich der dermatologische Nachwuchs und dessen Lehrer, die kostenfrei zu Unterrichtszwecken Bilder herunterladen können. Gewerbliche Nutzer werden gebeten, einen Obolus zu entrichten. Im Ganzen liegt für Fachinteressierte aus der Dermatologie und Industrie ein modernes Bildarchiv vor. Der Quizteil lädt zum Knobeln ein und macht mindestens soviel Spaß wie Günther Jauchs „Millionen-Quiz“.

Christiane Bayerl, Wiesbaden 